\title{
Source location and mechanism analysis of an earthquake triggered by the 2016 Kumamoto, southwestern Japan, earthquake
}

\author{
Takeshi Nakamura* ${ }^{*}$ and Shin Aoi
}

\begin{abstract}
The 2016 Kumamoto earthquake (Mw 7.0) occurred in the central part of Kyushu Island, southwestern Japan, on April 16, 2016. The mainshock triggered an event of maximum acceleration 700 gal that caused severe damage to infrastructure and thousands of homes. We investigate the source location of the triggered event, and the timing of large energy release, by employing the back-projection method for strong-motion network data. The optimal location is estimated to be [33.2750 $131.3575^{\circ}$ ] (latitude, longitude) at a depth of $5 \mathrm{~km}$, which is $80 \mathrm{~km}$ northeast of the epicenter of the mainshock. The timing is $33.5 \mathrm{~s}$ after the origin time of the mainshock. We also investigate the source mechanism by reproducing observed displacement waveforms at a near-source station. The waveforms at smallersized events, convolved with the source time function of a pulse width $1 \mathrm{~s}$, are similar to the signature of the observed waveforms of the triggered event. The observations are also reproduced by synthetic waveforms for a normal-fault mechanism and a normal-fault with strike-slip components at the estimated locations. Although our approach does not constrain the strike direction well, our waveform analysis indicates that the triggered earthquake occurred near the station that observed the strong motions, primarily via a normal-fault mechanism or a normal-fault with strike-slip components.
\end{abstract}

Keywords: 2016 Kumamoto earthquake, Triggered earthquake, Strong motion, Back-projection method, Green's function, Hypocenter determination, Source mechanism

\section{Background}

Two destructive earthquakes (named the 2016 Kumamoto earthquake) occurred in the central part of Kyushu Island, southwestern Japan (Fig. 1a), on April 14 and 15, 2016. The Japan Meteorological Agency (JMA) reported the moment magnitude and the source depth of the first and second mainshocks as 6.2 and $11.4 \mathrm{~km}$, and 7.0 and $12.4 \mathrm{~km}$, respectively. The mainshocks occurred in two active faults (Futagawa and Hinagu faults), predominantly via strike-slip mechanism with $\mathrm{E}-\mathrm{W}$ compression (Fig. 1a).

The fault model of the $M \mathrm{w} 7.0$ second mainshock (hereafter "mainshock"), evaluated from strong groundmotion data, showed rupture propagation in the

\section{*Correspondence: t_nakamura@bosai.go.jp}

National Research Institute for Earth Science and Disaster Resilience, 3-1 Tennodai, Tsukuba 305-0006, Japan northeast direction and maximum slip of $3.8 \mathrm{~m}$ at an epicentral distance of approximately $10-30 \mathrm{~km}$ (Kubo et al. 2016). Interferometric synthetic aperture radar (InSAR) data also showed large surface displacements at an epicentral distance of approximately $5-30 \mathrm{~km}$ in the northeast area (Ozawa et al. 2016). Hypocenter analysis of aftershocks indicated a linear distribution with total length of $50 \mathrm{~km}$ in the northeast direction from the epicenter of the mainshock (Yano and Matsubara 2016). In the off-fault area about $80 \mathrm{~km}$ northeast from the epicenter, the hypocenter distribution showed isolated seismic activities that are not directly continued to the main fault segments (Fig. 1a). Strong ground motions of more than 700 gal, which caused severe damage to infrastructure and thousands of homes (Oita prefecture, http:// www.pref.oita.jp/site/bosaiportal/280414jisin.html, last accessed on July 19, 2016), were observed in the area of the isolated activities (Aoi et al. 2016). The observed 
acceleration is inconsistently large compared with that estimated from the empirical relationship as a function of the hypocentral distance. In Fig. 1a, the colors indicate the anomalously large amplitude of acceleration in the isolated activity area.

We show seismic waveforms observed at strongmotion network stations of K-NET and KiK-net (Okada et al. 2004) operated by the National Research Institute for Earth Science and Disaster Resilience (NIED) in and around the source areas at the mainshock in Fig. $1 \mathrm{~b}$. $S$-wave propagation with apparent velocity of $3.5 \mathrm{~km} / \mathrm{s}$ is found at stations located off the source fault of the mainshock after $S$-wave propagation from the mainshock. In the high-frequency range $>20 \mathrm{~Hz}$ to suppress $S$ - and surface waves from the mainshock, the propagation of $P$-waves with apparent velocity of $6.3 \mathrm{~km} / \mathrm{s}$ is found at these stations (Fig. 1c). These phases start to propagate at $30-40 \mathrm{~s}$ after the origin time of the mainshock and differ from the coda phases of the mainshock. These observations suggest that an event might be triggered after the mainshock, possibly by external perturbations associated with the mainshock rupture such as stress changes to the area.

In this study, we analyze the source location and timing of seismic energy release for the triggered event by employing the back-projection method (Spudich and Cranswick 1984). In the waveform data, contaminations of coda waves from the mainshock into the onset of body waves from the triggered event are found at most stations, making it difficult to identify the onset. The backprojection method determines the event location and timing by evaluating coherent signals in stacked waveform and does not use the onset data. It is anticipated that the method may provide an alternative approach to estimating the source location and timing for such event data. We also reproduce observed waveforms of the triggered event by using waveforms of smaller-sized events as Green's functions and infer the source mechanism of the triggered event. We verify the estimated source location and mechanism by calculating synthetic waveforms. These source data investigated in this study would contribute to quantitatively studying the causes of observed strong motions, seismic activity around the triggered event, and stress transfer from the mainshock to the triggered event.

\section{Source location and timing of energy release for triggered event}

We employ the back-projection method (Spudich and Cranswick 1984) to investigate the hypocenter location and the timing of large energy release of the triggered event. For the investigation, we select 17 strong-motion stations of K-NET and KiK-net (white triangles in Fig. 1a; Additional file 1: Table S1) that record clear signals from the triggered event. Data from stations located in the northeastern and southwestern areas, which correspond to areas along the rupture direction, are not used. This is because there is significant contamination of mainshock coda waves within the frequency range of interest, resulting in significantly low signal-to-noise ratio.

The back-projection method has often been applied to investigate the rupture process using coherent phases stacked from high-frequency seismic waveforms observed in seismic array, without assuming source mechanisms and dimensions and without calculating synthetic waveforms (e.g., Ishii et al. 2005). The method has been also applied to investigate the hypocenter determination of events such as tremor activities (e.g., Kao and Shan 2004) and micro-events (e.g., Vlček et al. 2015). By enhancing the coherency of stacked waveforms, the method might be appropriate for analyzing waveform data contaminated by coda waves of the mainshock as the present case, in which it is difficult to identify the onset of body waves. We also suppose that the estimates obtained via this approach will complement those of the conventional hypocenter determination approach.

We apply the method to the $S$-wave component of envelope waveforms. We integrate acceleration data of the horizontal component within the frequency range $3-8 \mathrm{~Hz}$ and produce the vector sum of horizontal data from the envelope of each component using the Hilbert transform. We then calculate the mean amplitude every $0.1 \mathrm{~s}$ within a moving time window of $0.2 \mathrm{~s}$. The obtained smoothed envelope waveform is normalized

(See figure on next page.)

Fig. 1 Location map of the 2016 Kumamoto earthquake and strong-motion records. a Epicenter (yellow star) and source mechanism (beach ball) of the 2016 Kumamoto earthquake occurred on April 15, 2016, and aftershocks (gray dots) occurred within $24 \mathrm{~h}$. White circles and triangles indicate K-NET and KiK-net strong-motion stations and stations used for the back-projection analysis, respectively. Circles, which are located in the direction of $\mathrm{N}^{\circ} 5^{\circ}$ from the epicenter of the mainshock, indicate stations of strong-motion waveforms shown in (b) and (c). The Japanese Islands and the focal area are shown in the top-left inset. $\mathbf{b}$ North-south component of acceleration waveforms. Black and red traces show waveforms at K-NET and KiK-net stations and K-NET station OIT009, respectively. Station codes are shown in the right side of each trace. Thick gray lines indicate an identified seismic phase different from phases of the mainshock, and S-wave propagation of apparent velocity $3.5 \mathrm{~km} / \mathrm{s}$ from station OIT009. c Vertical component of $20 \mathrm{~Hz}$ high-pass filtered acceleration waveforms. Thick gray lines indicate an identified seismic phase different from phases of the mainshock, and $P$-wave propagation of apparent velocity $6.3 \mathrm{~km} / \mathrm{s}$ from station OIT009 
a $35^{\circ}$

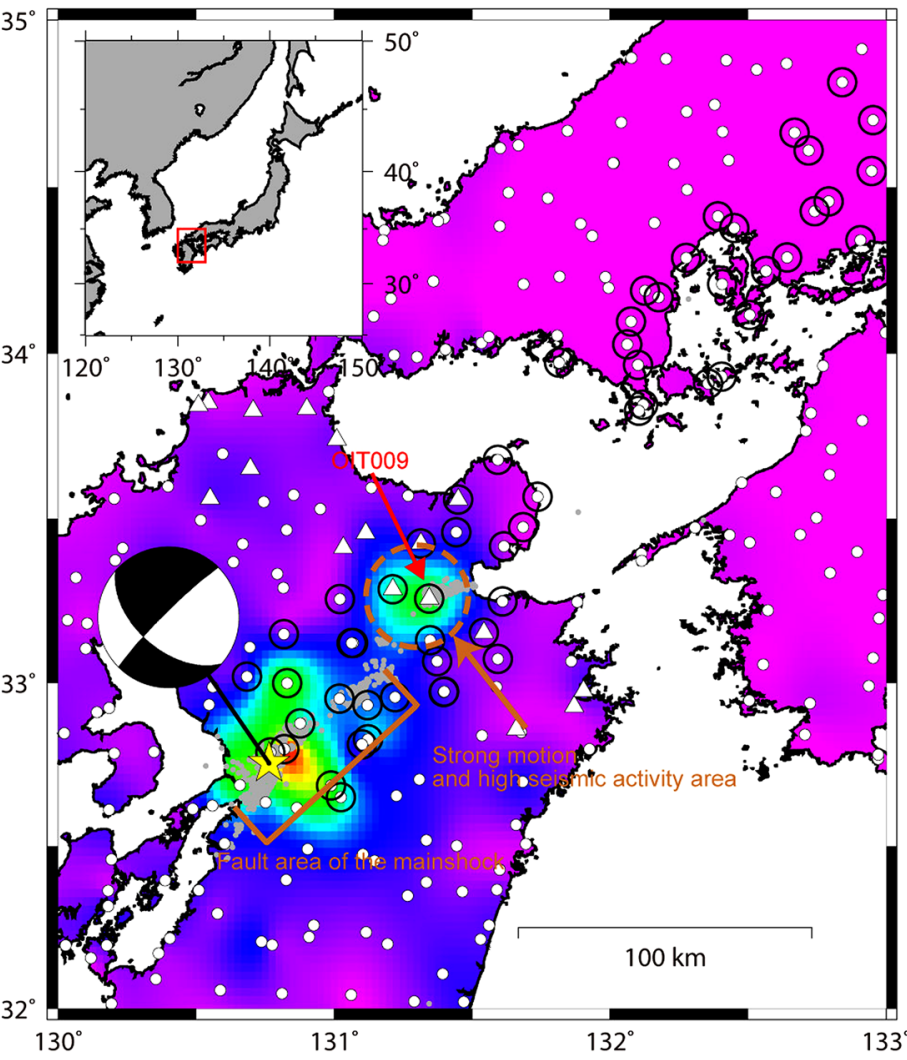

Mainshock

Station (K-NET, KiK-net)

- Events after the mainshock

$\triangle$ Station used in back projection analysis

$\bigcirc$, () Station used for waveform plot in Figures 1(b) and (c)

Max. acc. (cm/s/s)

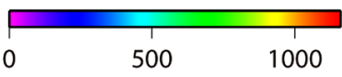

Waveform at station OIT009 Waveform at other stations Time (s)

b

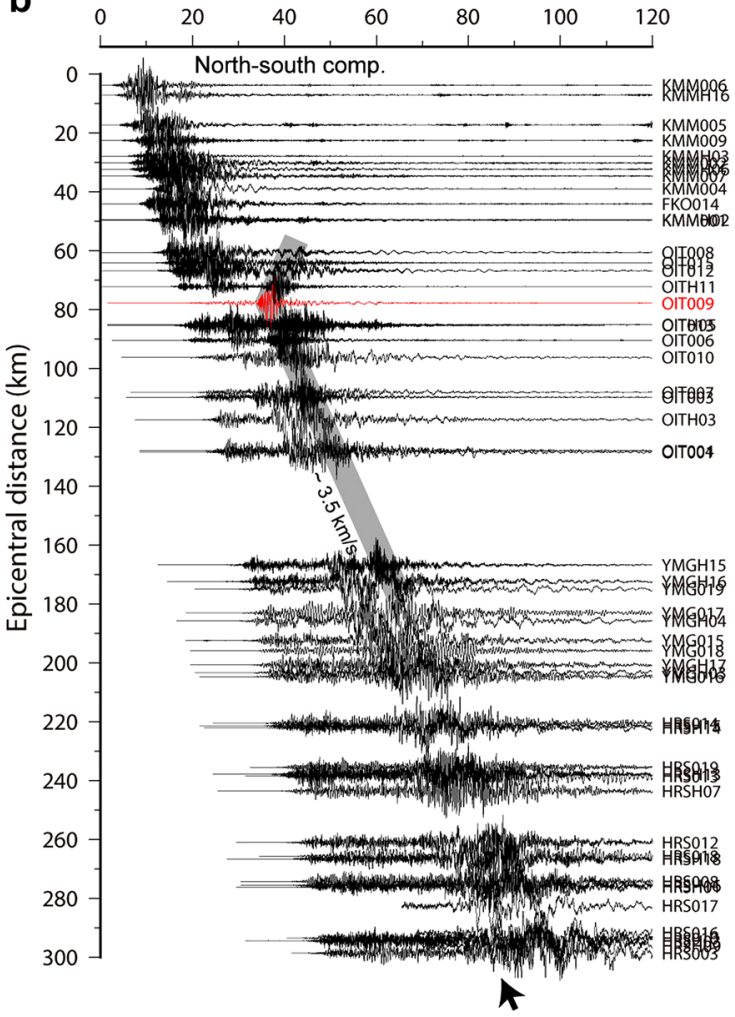

S-wave from mainshock c

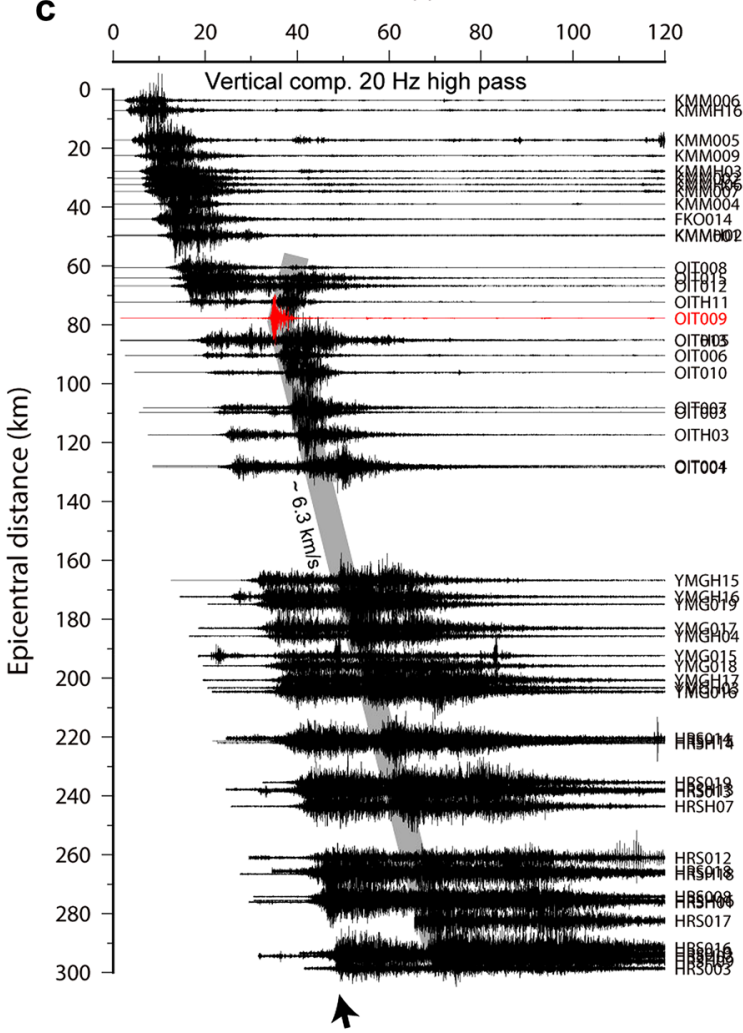

P-wave from mainshock 
by the maximum amplitude of the phase of the triggered event at each trace. The reason for the frequency range of $3-8 \mathrm{~Hz}$ is that we aim to suppress coda waves from the mainshock, by high-pass filtering in a corner frequency of $3 \mathrm{~Hz}$, and to avoid using complex waveforms including $P$-wave and other high-frequency components from the triggered event, by low-pass filtering in a corner frequency of $8 \mathrm{~Hz}$.

We stack the envelope waveforms for an assumed source location and timing of energy release using the following equation, which basically replicates that proposed by Kao and Shan (2004):

$S_{i}(t)=\frac{1}{N} \sum_{n=1}^{N}\left\{\frac{1}{2 M} \sum_{m=-M}^{M-1} A_{n}\left(t+T_{i, n}+m \Delta t\right)\right\}$,

where $S_{i}$ is the stacked waveform for the source grid $i$ as a function of the time $t$ for large energy release of the triggered event, $A_{n}$ is the envelope waveform at station $n, \Delta t$ is the time interval of data sampling, $T_{i, n}$ is the travel time of $S$-wave from the source grid $i$ to station $n, M$ is the number of time points within the half-length of the time window, and $N$ is the number of stations. For travel-time calculations, we use the one-dimensional (1D) velocity structure model that is routinely used by Kyushu University to determine hypocenters in this area (Fig. 2). We incorporate station corrections into the calculated times to correct the travel times induced by lateral variations in the three-dimensional (3D) velocity structure. The corrections are obtained from travel-time residuals that are estimated from the hypocenter determinations for 76 events that occurred within our study area (Step 1 in Fig. 3a; Table 1) since 1996. The travel-time calculations do not utilize apparent velocity, because of the difficulty, at near-source stations, in assuming incident plane waves from the horizontal direction. We identify the optimal grid point in time and space from the largest amplitude of the stacked waveform. By the stacking analysis of using multiple station data, we suppress the local site effect at a station for the estimation.

The grid sizes and search regions used in this study are summarized in Table 1 . We conduct multi-scale analysis to efficiently obtain the optimal solution: We first search for the optimal solution for a rough grid size across a wide area in Step 1 (see Fig. 3a; Table 1) and then search for the next solution for a finer grid size in a smaller area in Step 2 (see Fig. 3a; Table 1). The smallest grid in the horizontal direction for the search is $0.0125^{\circ}$ (Step 4 in Fig. 3a; Table 1), which is comparable to the maximum wavelength for our use of waveforms.

We show estimated results for the triggered event by the back-projection method in Fig. 3b-d. Since the

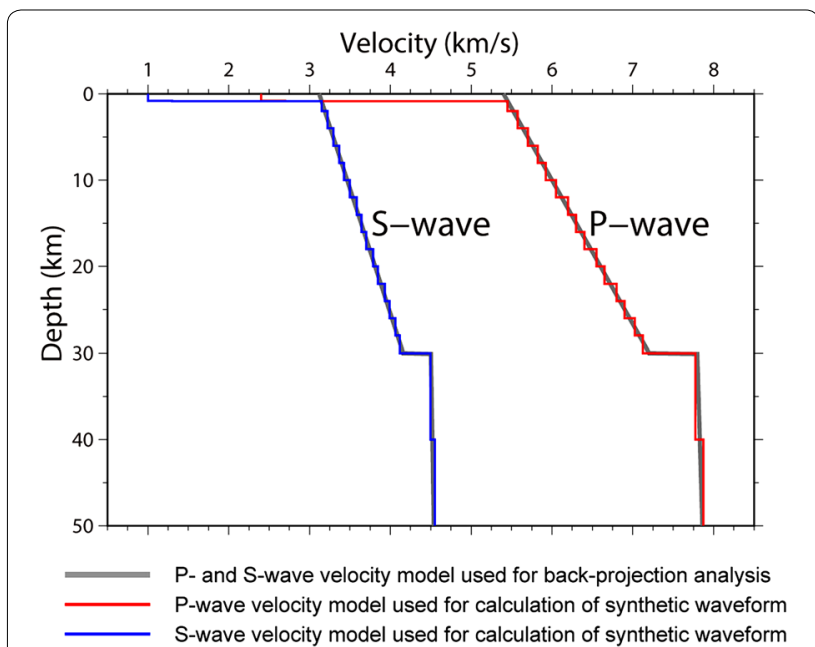

Fig. 2 Velocity structure models used in this study. Gray thick lines indicate $P$ - and $S$-wave structure models used for the back-projection analysis. The model is used by Kyushu University for routine analysis of hypocenter determination in this area. Red and blue lines indicate $P$ - and S-wave structure models used for calculating synthetic waveforms. The model is composed of the velocity structure obtained by discretization of the structure model by Kyushu University, and the velocity structure of sediment layers around station OIT009 by Koketsu et al. (2008)

stations used are distributed in a NW-SE direction, Fig. 3b-d shows large stacked amplitudes in a spatially linear distribution in the NE-SW direction, indicating a low resolution in this direction for our analysis relative to that in the NW-SE direction. The optimal solution for the epicenter of the large energy release and the depth for the triggered event is latitude $33.2750^{\circ}$, longitude $131.3575^{\circ}, 5 \mathrm{~km}$, respectively. The best solution of the source is located in the northeastern area of K-NET station OIT009. The timing of the energy release is $33.5 \mathrm{~s}$ after the origin time (16:25:05 UTC, on April 15, 2016) of the mainshock. The second optimal solution is found in the northern area of station OIT009 (dashed red circle in Fig. $3 \mathrm{~b}-\mathrm{d}$ ). The hypocenter is located at latitude $33.2875^{\circ}$, longitude $131.3325^{\circ}$, at a depth of $5 \mathrm{~km}$, and the timing of the solution is $33.8 \mathrm{~s}$. We checked the performance of our analysis using data from well-relocated events that occurred in the area of Step 4 and are used for analyzing travel-time residuals, and evaluated the estimation error by comparing the estimated hypocenters with those listed in the JMA hypocenter catalogue. The standard errors are 4.6 and $4.7 \mathrm{~km}$ for the horizontal and depth directions, respectively. The error for time is $1.4 \mathrm{~s}$.

We here address that the obtained results for time and space using high-frequency waveform data do not match the origin time of the event and the initial break point but 

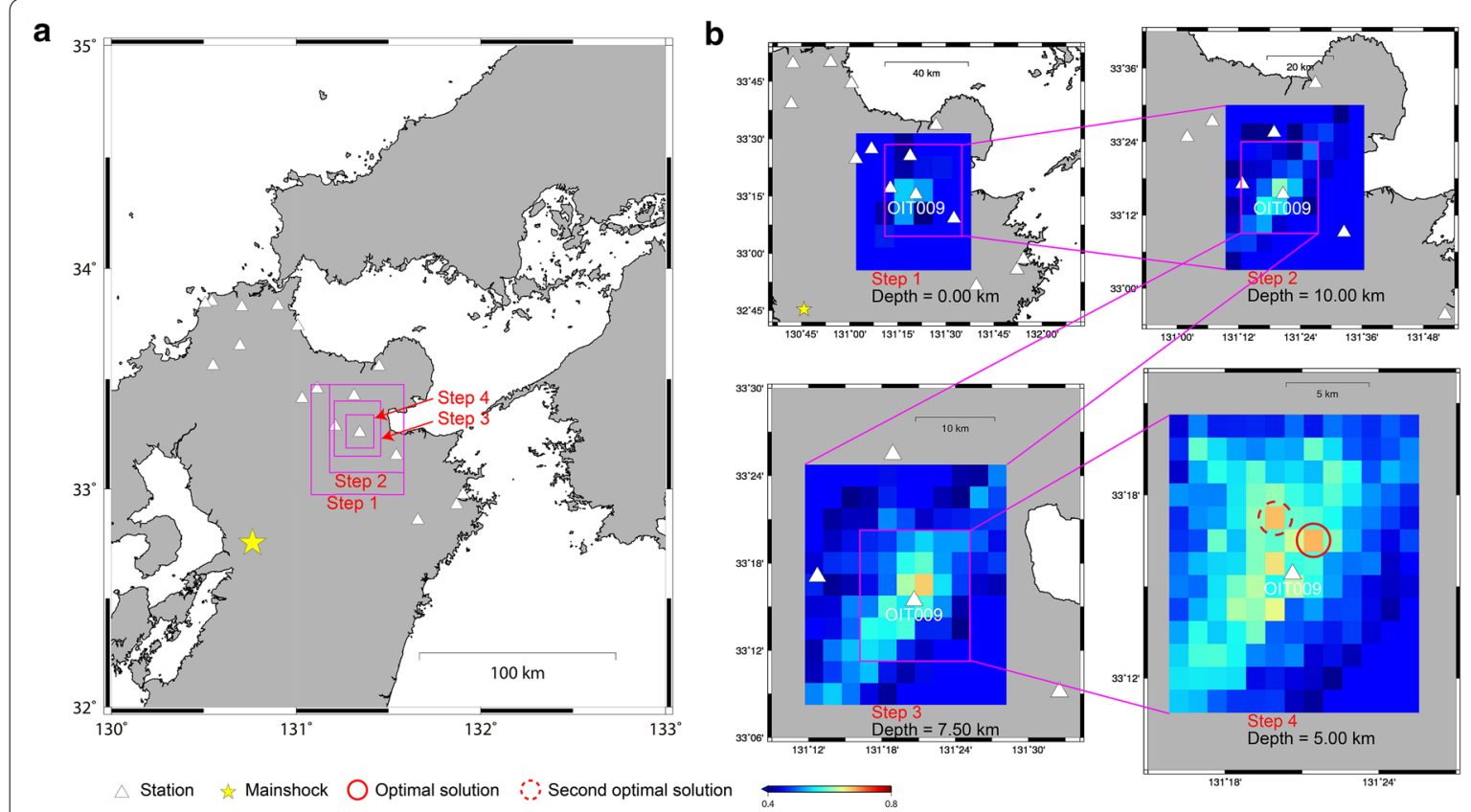

C
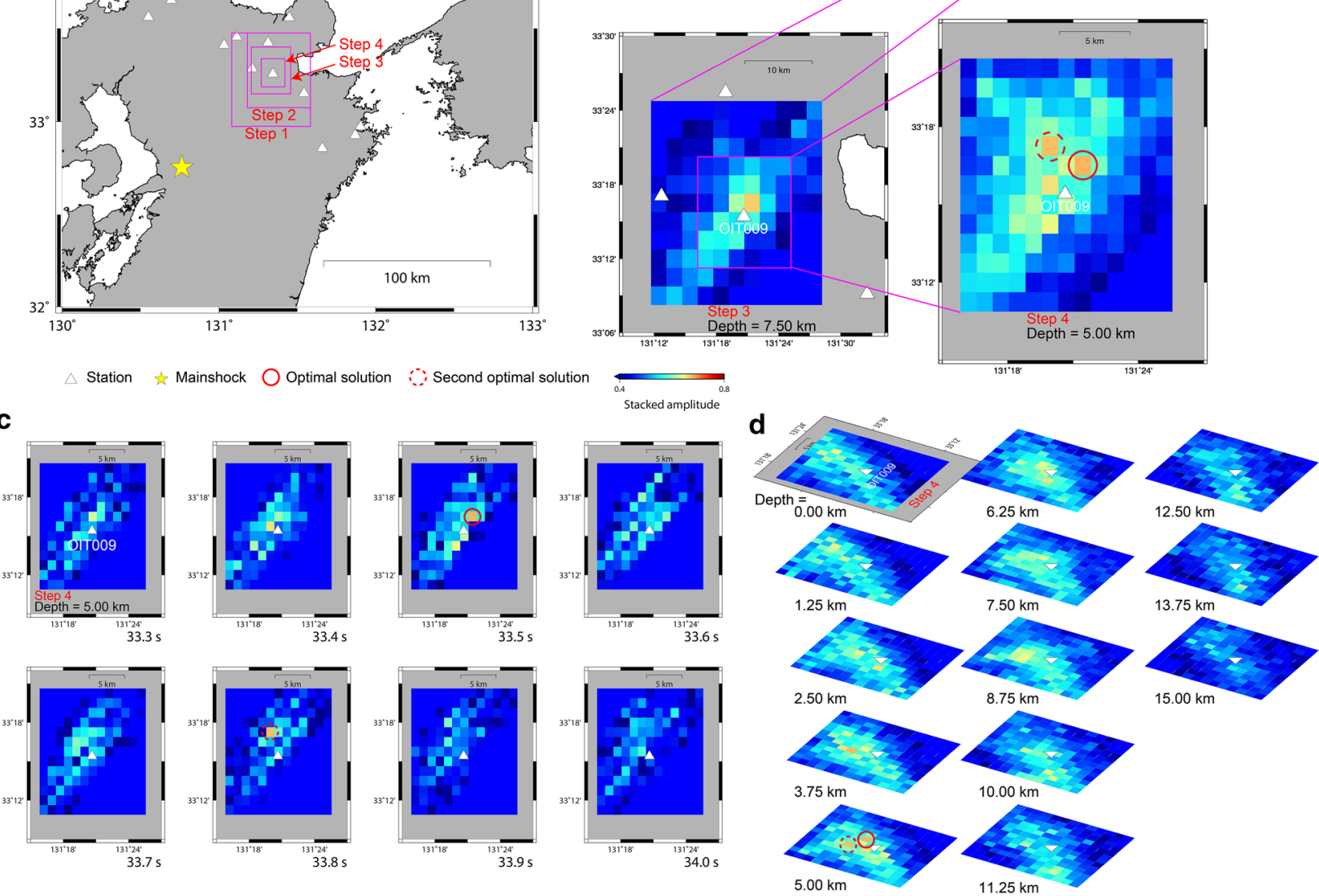

Fig. 3 Hypocenter and origin time of the triggered event estimated by the back-projection method. a Areas used for the multi-scale analysis. A yellow star indicates the epicenter of the mainshock. White triangles indicate stations used in the analysis. $\mathbf{b}$ Epicenter of the triggered event obtained from multi-scale analysis. Color at each grid indicates the maximum stacked amplitude within the time range in Table 1. A red and a dashed red circles indicate the optimal and the suboptimal result for the epicenter of the triggered event, respectively. Panels show results for the distribution of the stacked amplitude for spatial grid intervals of $0.1^{\circ}, 0.05^{\circ}, 0.025^{\circ}$, and $0.0125^{\circ}$. Each panel shows the distribution at the depth of the optimal result. c Distribution of the stacked amplitude at an elapsed time of 33.3-34.0 s from the origin time of the mainshock. The distribution shows the result for the grid interval of $0.0125^{\circ}$ at the depth of $5 \mathrm{~km}$. $\mathbf{d}$ Distribution of the maximum stacked amplitude within the time range for the grid interval of $0.0125^{\circ}$ at each depth

instead indicate the timing of large high-frequency energy release and the released area. The source studies for highfrequency seismic radiation (e.g., Spudich and Frazer 1984) showed that the radiated energies are generated by changes in slip and/or rupture speed, and the initiation of large slips. Following their results, the estimated results in our analysis may correspond to the source of large highfrequency energy release in and around a large slip region.
We show the observed waveforms sorted in the epicentral distance based on the estimated epicentral location of the triggered event and the stacked waveform in Fig. 4, which ensures agreement of the predicted arrival time with the phase of the triggered event at the stations (Additional file 1: Figure S1) and enhances the coherency by appropriately stacking the phase. The amplitude of the stacked waveform for the phase of the triggered event is 
Table 1 Spatial grid size and search area and time for the back-projection analysis

\begin{tabular}{|c|c|c|c|c|c|}
\hline $\begin{array}{l}\text { Step } \\
\text { number }\end{array}$ & $\begin{array}{l}\text { Grid size } \\
\text { in horizontal } \\
\text { direction }\left(^{\circ}\right)\end{array}$ & $\begin{array}{l}\text { Area for grid search } \\
\text { (latitude/longitude) }\end{array}$ & $\begin{array}{l}\text { Grid size } \\
\text { in vertical } \\
\text { direction }(\mathrm{km})\end{array}$ & $\begin{array}{l}\text { Depth range } \\
\text { for grid } \\
\text { search }(\mathrm{km})\end{array}$ & $\begin{array}{l}\text { Time range } \\
\text { for grid } \\
\text { search (s) }\end{array}$ \\
\hline 1 & 0.1 & N32.9750-33.4750/E131.0825-131.5825 & 10 & $0-30$ & $30-40$ \\
\hline 2 & 0.05 & N33.0750-33.4750/E131.1825-131.5825 & 5 & $0-20$ & $32-40$ \\
\hline 3 & 0.025 & N33.1500- 33.4000/E131.2075-131.4575 & 2.5 & $0-15$ & $32-38$ \\
\hline 4 & 0.0125 & N33.1875-33.3375/E131.2700-131.4200 & 1.25 & $0-15$ & $32-35.5$ \\
\hline
\end{tabular}

0.68. In Fig. 4, phases with large amplitude before $30 \mathrm{~s}$ are from the mainshock.

\section{Possible source mechanism inferred from single-station data}

From the F-net moment tensor (MT) database operated by NIED, we find that normal-fault events have occurred in and around the source area of the triggered event, which is consistent with geological survey findings that active faults in this area are primarily the normal-fault type (e.g., Kamata 1989). However, the mechanism of the triggered event has not been determined, since source mechanism analyses (such as analyses of the $P$ waveform similarities between events, the polarity of initial $P$-wave, the particle motion of initial $S$-wave, and the waveform inversion for surface waves) are difficult because of the contamination of coda waves from the mainshock at most of stations. The iterative deconvolution method (Kikuchi and Kanamori 1991) that can decompose the phase of multiple subevents and estimate their mechanism is also difficult in the present case, because of significant differences in signal level in the low-frequency range between the mainshock and the triggered event.

We here reproduce the waveform of the triggered event from those of smaller-sized events (Mw 4.1-5.1 as listed in Table 2) around station OIT009 in order to infer the mechanism. We assume the observed waveform at the smaller events as a Green's function of the triggered event. We convolve such waveforms with a cosine-type source time function and compare the convolved waveforms with waveforms of the triggered event, which is a similar approach to reproducing the waveforms of a large earthquake by using the empirical Green's function (Hartzell 1978).

We use the horizontal component of high-pass filtered displacement waveforms in a corner frequency of $0.3 \mathrm{~Hz}$ for the convolution. The reason for focusing on this frequency is that the spectrum of the phase from the triggered event at station OIT009 shows a dominant frequency of approximately $0.3-2 \mathrm{~Hz}$, which may indicate the dominant frequency of the source spectrum. Another reason is that the phase from the triggered event at frequencies $>0.3 \mathrm{~Hz}$ is found to have larger amplitudes than "noise", which are coda waves from the mainshock. For the other stations, the phase from the triggered event is not clearly found within this frequency range in the displacement or is significantly disturbed by coda waves of the mainshock.

The waveforms for Green's function are ones at events that occurred at shallow depth $<20 \mathrm{~km}$ and in the epicentral area at a distance $<10 \mathrm{~km}$ around station OIT009. We show the source mechanism, magnitude, and epicenter location of five smaller events used for the analysis in Table 2 and Fig. 5, which are listed in the F-net MT database. The source mechanism of all five events shows normal-fault type or normal-fault with strike-slip component. The strike for events on January 1, 1999 (Event 1) and April 29, 2016 (Event 5) is $\mathrm{N} 81^{\circ} \mathrm{E}$ and $\mathrm{N} 117^{\circ} \mathrm{E}$, respectively, and for the other events is approximately $\mathrm{N} 90^{\circ} \mathrm{E}$. The magnitudes are $\mathrm{Mw}$ 4.1-5.1. Four events occurred within 2 weeks after the triggered event in the northeastern area of station OIT009 near the estimated epicenter of the triggered event, and may be aftershocks of the triggered event. We use the cosine-type source time function for convolution of waveforms for these five smaller events. By trial and error (comparing the waveform of the triggered event with the convolved waveform), we obtain $1 \mathrm{~s}$ as the optimal pulse width of the function.

We show the waveform comparisons in Fig. 6. For the vertical component, the waveform amplitude of the triggered event is small compared with that of the horizontal component and is contaminated by coda waves from the mainshock. The amplitude ratio of vertical component/ horizontal component in the convolved waveform for the five smaller events is relatively large and does not explain the ratio in that of the triggered event. The amplitude ratio at a station just near the source can be significantly changed by the slight differences in mechanism and the location between two events, which may account for the discrepancy found in the components.

For the north-south component, we find a slight time shift of the initial $S$-phase between the triggered event and Events 1 and 4. Another difference between the triggered event and Event 3 is found in amplifications of later phases after the initial $S$-phase, which may 


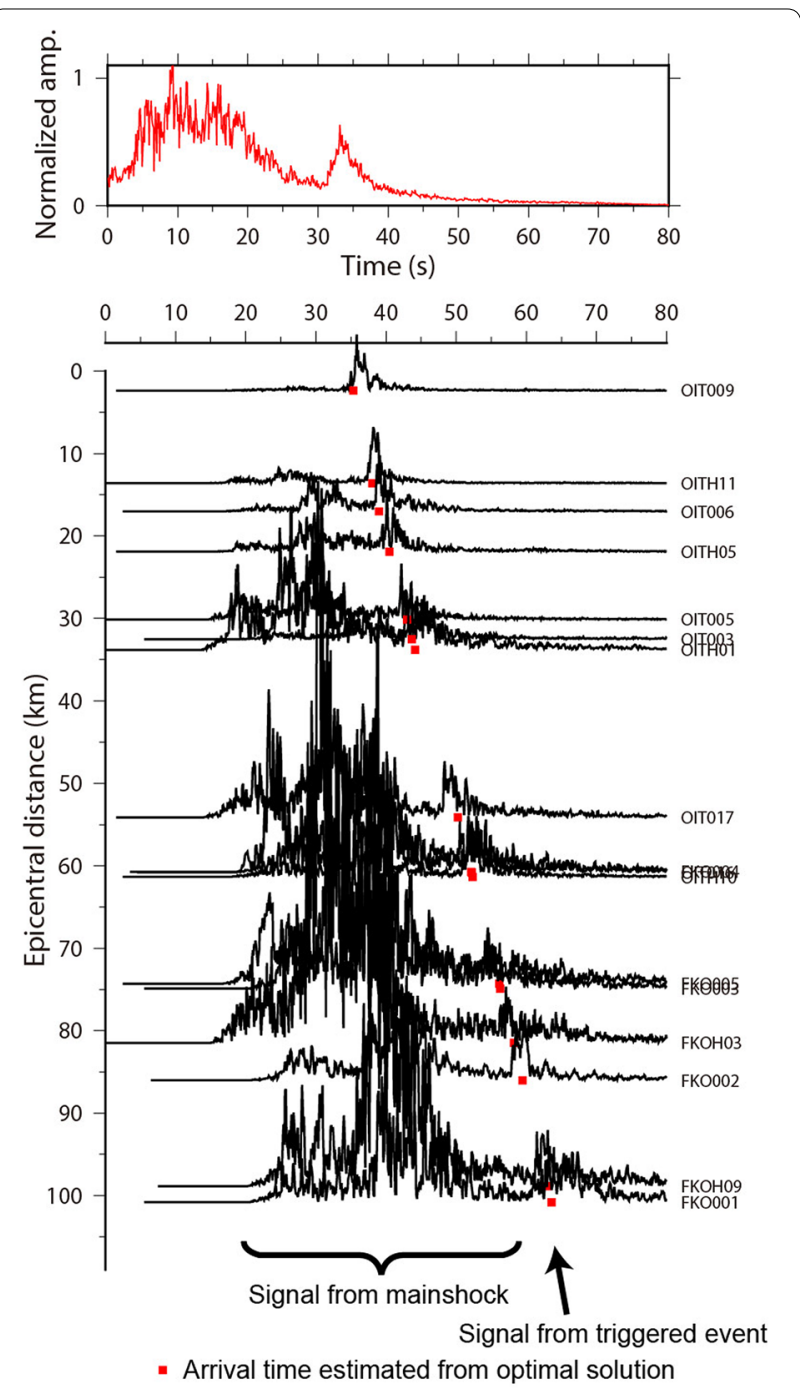

Fig. 4 Stacked and envelope waveforms. Red traces in the upper panel show the waveform stacked from envelope waveform based on the travel time estimated from the optimal result for the source location of the triggered event. The time $t=0$ on the horizontal axis is the origin time of the mainshock. Phases with large amplitudes before $30 \mathrm{~s}$ are the stacked phases of the mainshock. Black traces in the lower panels show envelope waveforms at strong-motion stations. The traces are sorted by epicentral distance based on the optimal result for the source location. Red squares indicate arrival times estimated from the optimal solution for the hypocenter of the triggered event be amplified not by the source and the path effects but by the effect of sediment layers at shallow depth, since monochromatic signals in the amplified later phases are found. For the east-west component, the initial $S$-phase with large amplitudes is reproduced well from the convolved waveforms for the five smaller events. For Events $2-5$, the second phase of the triggered event (appearing $2 \mathrm{~s}$ after the initial phase) is also reproduced. The reproduction is also verified for convolved waveforms from a small $M \mathrm{w} 3.7$ event of the strike-slip type with a slight normal-fault component (Additional file 1: Figure S2) that was rapidly determined by waveform analysis and listed in the AQUA CMT database (Matsumura et al. 2006), indicating that the use of intermediate-sized earthquakes $(M \mathrm{w}$ 4.1-5.1) in this analysis is not inappropriate for Green's function. However, the second phase is not reproduced by the convolved waveform of only Event 1 . Of the five smaller-sized events, the phase in the horizontal component of the convolved waveform for Event 5 is likely to match that of the triggered event. Although we cannot determine the fault parameters from our waveform comparisons, we speculate that the source may be located in the northeastern area, near station OIT009, and be a normal-fault type or a normalfault with strike-slip components.

The maximum amplitude of the high-pass filtered waveform in a corner frequency of $0.3 \mathrm{~Hz}$ for the triggered event is $13 \mathrm{~cm}$, which is $17-340$ times larger than that of the convolved waveform for the smaller events. By referring to the moment magnitude for smaller events listed in the F-net MT database, the magnitude of the triggered event is then roughly estimated to be $M w 5.3$ and $M w$ 5.7-5.9 in the case of using Event 5 and the other Event data, respectively. However, we should note that this estimation assumes only minor differences in source mechanism and location between the triggered and the smaller-sized events. Furthermore, this estimation significantly depends on the pulse width of the source time function used for calculating the convolution. Using a pulse width of $2 \mathrm{~s}$ for the convolution, the estimated magnitude could range from $M w$ 5.5-6.1.

Table 2 Source location and double-couple mechanism for smaller-sized events around station OIT009 from the F-net MT database

\begin{tabular}{|c|c|c|c|c|c|c|c|}
\hline Origin time (UTC yyyy/mm/dd, hh:mm:ss) & Latitude $\left({ }^{\circ}\right)$ & Longitude $\left({ }^{\circ}\right)$ & Depth $(\mathrm{km})$ & $M w$ & Strike $\left(^{\circ}\right)$ & $\operatorname{Dip}\left({ }^{\circ}\right)$ & Rake $\left(^{\circ}\right)$ \\
\hline 1999/01/01, 14:30:04 & 33.3112 & 131.3468 & 8 & 4.2 & 81 & 50 & -68 \\
\hline 2016/04/15, 22:11:37 & 33.2715 & 131.3957 & 5 & 5.1 & 96 & 61 & -97 \\
\hline 2016/04/15, 23:27:00 & 33.2817 & 131.3900 & 5 & 4.2 & 95 & 70 & -80 \\
\hline 2016/04/16, 14:26:24 & 33.2752 & 131.3752 & 5 & 4.1 & 85 & 65 & -99 \\
\hline 2016/04/29, 06:09:34 & 33.2578 & 131.3680 & 5 & 4.5 & 117 & 74 & -64 \\
\hline
\end{tabular}




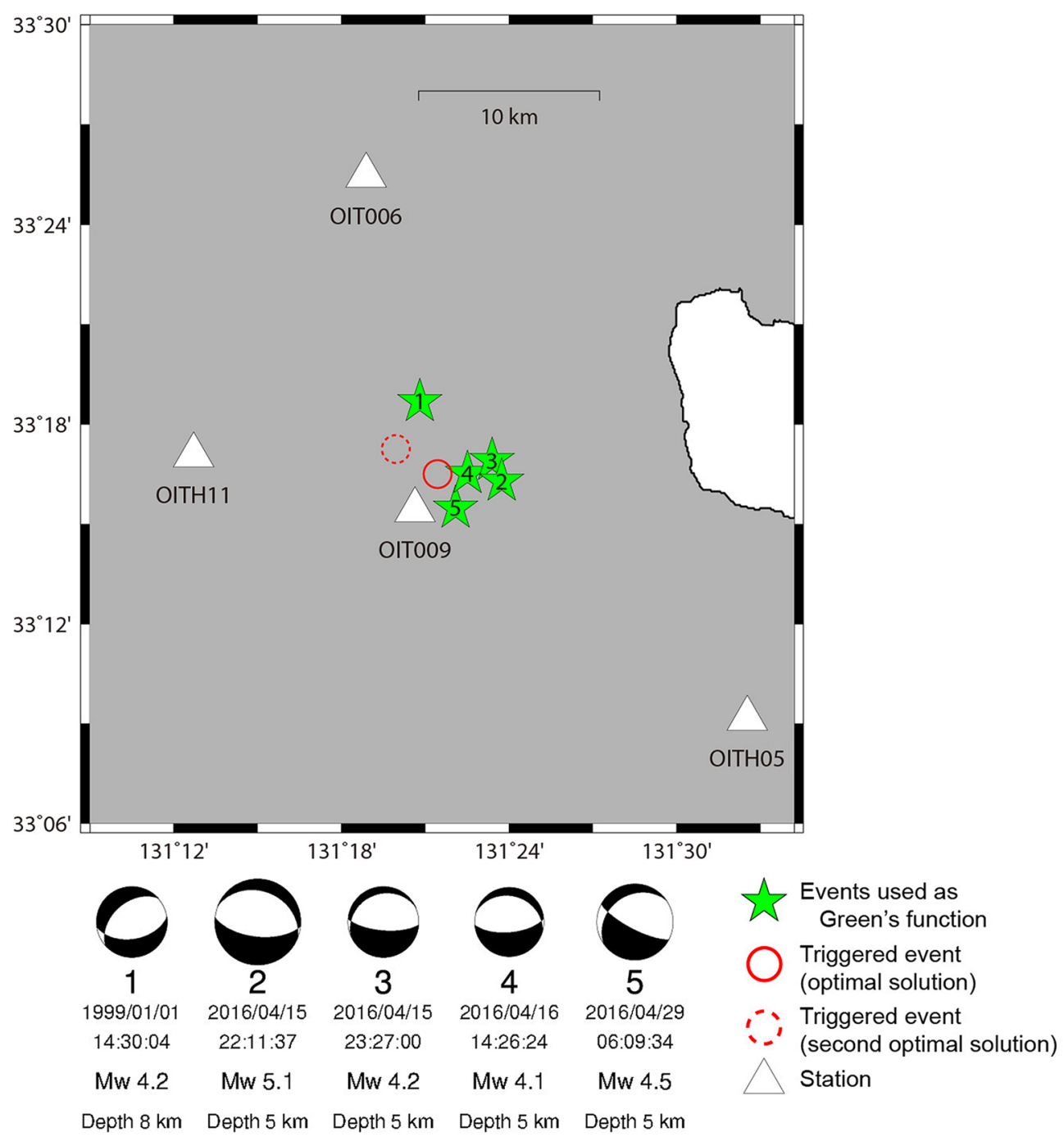

Fig. 5 Source location and mechanism of smaller-sized events around station OIT009. A red and a dashed red circles indicate the optimal and the suboptimal result for the epicenter of the triggered event, respectively. White triangles indicate the locations of K-NET and KiK-net stations. Green stars indicate the source locations of smaller events. Double-couple mechanisms, origin times, and magnitudes of the smaller events are shown at the bottom

\section{Discussion}

Our back-projection and convolution analyses indicate that the source is located near station OIT009 and that the mechanism is a normal-fault type or a normal-fault with strike-slip components. However, the smaller events have nearly the same mechanism. We here calculate synthetic waveforms for the source location estimated by the back-projection method by using various types of the mechanism from a theoretical approach and investigate the possibility of other types of source mechanism.

We employ the discrete wavenumber method (Nakamura and Takenaka 2006) for the waveform calculation by using a 1D velocity model (red and blue lines in Fig. 2) composed of the velocity structure obtained by the discretization of the model used in the back-projection analysis (gray thick line in Fig. 2) and the velocity structure of sediment layers in this area by Koketsu et al. (2008). We use the same cosine-type source time function of the pulse width of $1 \mathrm{~s}$ as that used in the previous section. The seismic moment used is $M_{0}=10^{13} \mathrm{Nm}$. Assuming the double-couple source, we calculate waveforms for the source location estimated in the backprojection analysis by using various source mechanisms with fault parameters within the range: strike direction $0^{\circ}-360^{\circ}$, dip direction $0^{\circ}-90^{\circ}$, and rake angle of $-180^{\circ}$ to $180^{\circ}$. We calculate 13,690 cases by varying these parameters every $10^{\circ}$ within the given ranges. We also calculate waveforms for the source location and mechanisms of 


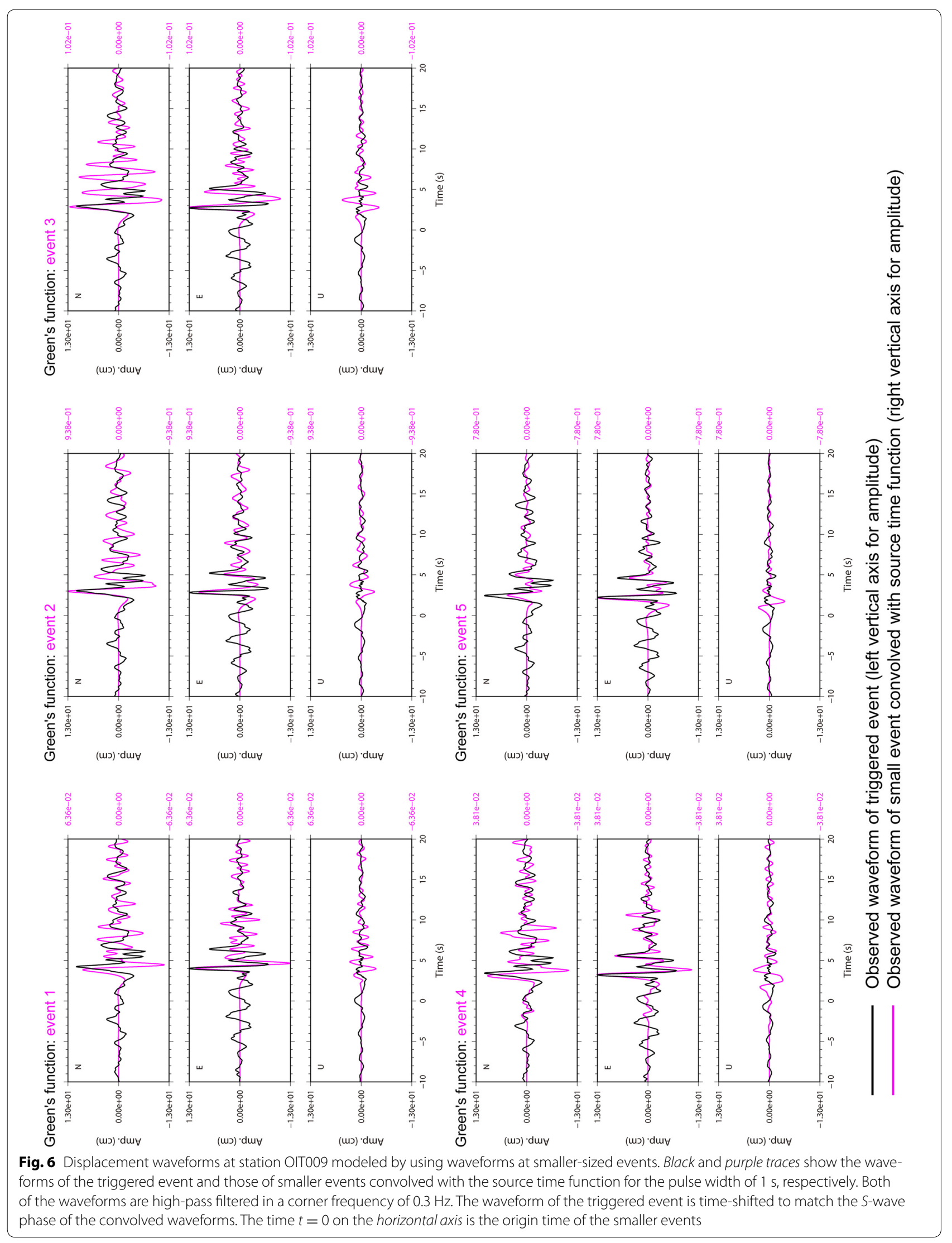


Events 1-5 (Fig. 5). We evaluate the fitting between synthetic and observed waveforms by the variance reduction VR (\%) based on the following equation, which is generally used in moment tensor analysis (e.g., Matsumura et al. 2006),

$$
\mathrm{VR}=\left(1-\frac{\sum_{i} \int\left(s_{i}(t)-o_{i}(t)\right)^{2} \mathrm{~d} t}{\sum_{i} \int\left(o_{i}(t)\right)^{2} \mathrm{~d} t}\right) \times 100,
$$

where $s_{i}$ and $o_{i}$ are the synthetic and observed waveforms of the $i$ th component, respectively. The variance reduction VR $=100 \%$ indicates complete fitting between the waveforms. We use the amplitude normalized by the maximum one of the horizontal components, because the moment magnitude and the seismic moment of the triggered event for calculation of the synthetic waveform are unknown and are not estimated in this step. We use the three-component waveforms to calculate the variance reduction. For the vertical component, the waveform amplitude is multiplied by a low weighing factor because evaluation is made difficult by the significant contamination by coda waves from the mainshock into the component. Both the observed and synthetic waveforms are displacements and high-pass filtered in a corner frequency of $0.3 \mathrm{~Hz}$. The length of the time window is $4 \mathrm{~s}$ from the arrival time of $S$-waves. Because arrival times are affected by three-dimensional heterogeneous structures, the time of synthetic waveforms is shifted within a range of -0.5 to $0.5 \mathrm{~s}$. We take the variance reduction of the maximum value in the shifted time range as the estimated results. In this source analysis, we assume that the hypocenter estimated by the back-projection method is located near the large slips. By simplifying the source as the point source, we infer the mechanism type of the triggered event as a first-order approximation.

Figure 7 shows the comparison of the observed waveform of the triggered event with the synthetic waveforms. As we find the same features in Fig. 6, the phase of the horizontal component in the synthetic waveform for Event 1 is shifted to that of the observation, which is not found in the phase for Events 2-5. The second phase of the triggered event for the east-west component is also not reproduced. Because of this, the low variance reduction of $-65 \%$ is obtained for Event 1 . The synthetic waveforms of the horizontal component for the other Events appear to explain the observation. By searching the mechanism in the case of the source location estimated by the back-projection analysis, the optimal one is (strike $200^{\circ}$, dip $30^{\circ}$, rake $-100^{\circ}$ ); note that these values of the angles may not be uniquely constrained due to errors caused by the assumption of the point source and limitations due to the use of only single-station data in the analysis. The synthetic waveform for the mechanism of the optimal solution determined by evaluating the variance reduction is shown in the bottom right of Fig. 7. The synthetic waveform explains the observations with the variance reduction of $57 \%$, indicating good agreement between the waveforms. The magnitude estimated from the comparison of the synthetic waveform amplitude with that of the observed one is $M w 5.6$, although this estimation may be overestimated because of amplifications in the observed waveform affected by coda waves from the mainshock.

We plot the estimated variance reduction for the source location estimated by the back-projection analysis for various mechanisms on the triangle diagram of the focal mechanism (Frohlich 1992) in Fig. 8a, which shows the top 100 results of the mechanism with high variance reduction from the 13,690 cases. The distribution of the source mechanism indicates that the normal-fault are probable mechanisms to explain the observations, although we cannot constrain the strike direction, which is presented as nearly invariant for the strike angle in Additional file 1: Figure S3, which shows the comparison of the distribution of variance reductions as a function of the fault parameters. We also plot the estimated variance reduction from the hypocenter of the second optimal solution (Fig. 2b) located in the northern area of station OIT009, indicating that a normal-fault type with strikeslip components is a probable mechanism. In contrast, the opposite of the normal-fault and the normal-fault with strike-slip component, i.e., the compression type, does not show the high variance reduction for either of the hypocenters (Fig. 8a, b) and is unlikely to explain the observations from the synthetic waveforms. These investigations suggest that the triggered event might occur near station OIT009 via a normal-fault mechanism or a normal-fault with strike-slip components.

\section{Conclusions}

We investigated the source location and mechanism of an event triggered during the 2016 Kumamoto earthquake (Mw 7.0) on April 16, 2016. We applied the back-projection method for velocity waveforms within the frequency range $3-8 \mathrm{~Hz}$. The optimal location is estimated to be $\left[33.2750^{\circ}, 131.3575^{\circ}\right]$ (latitude, longitude) at a depth of $5 \mathrm{~km}$, which is located just near the station that shows the peak acceleration amplitude of 700 gal during the event. The timing of large energy release of the triggered event is estimated to be $33.5 \mathrm{~s}$ after the origin time of the mainshock. We reproduced observed waveforms of high-pass filtered displacements in a corner frequency of $0.3 \mathrm{~Hz}$ at the station by using waveforms at smallersized events convolved with the source time function for the mechanism analysis. The convolved waveforms for the smaller events show agreement with the waveform of 


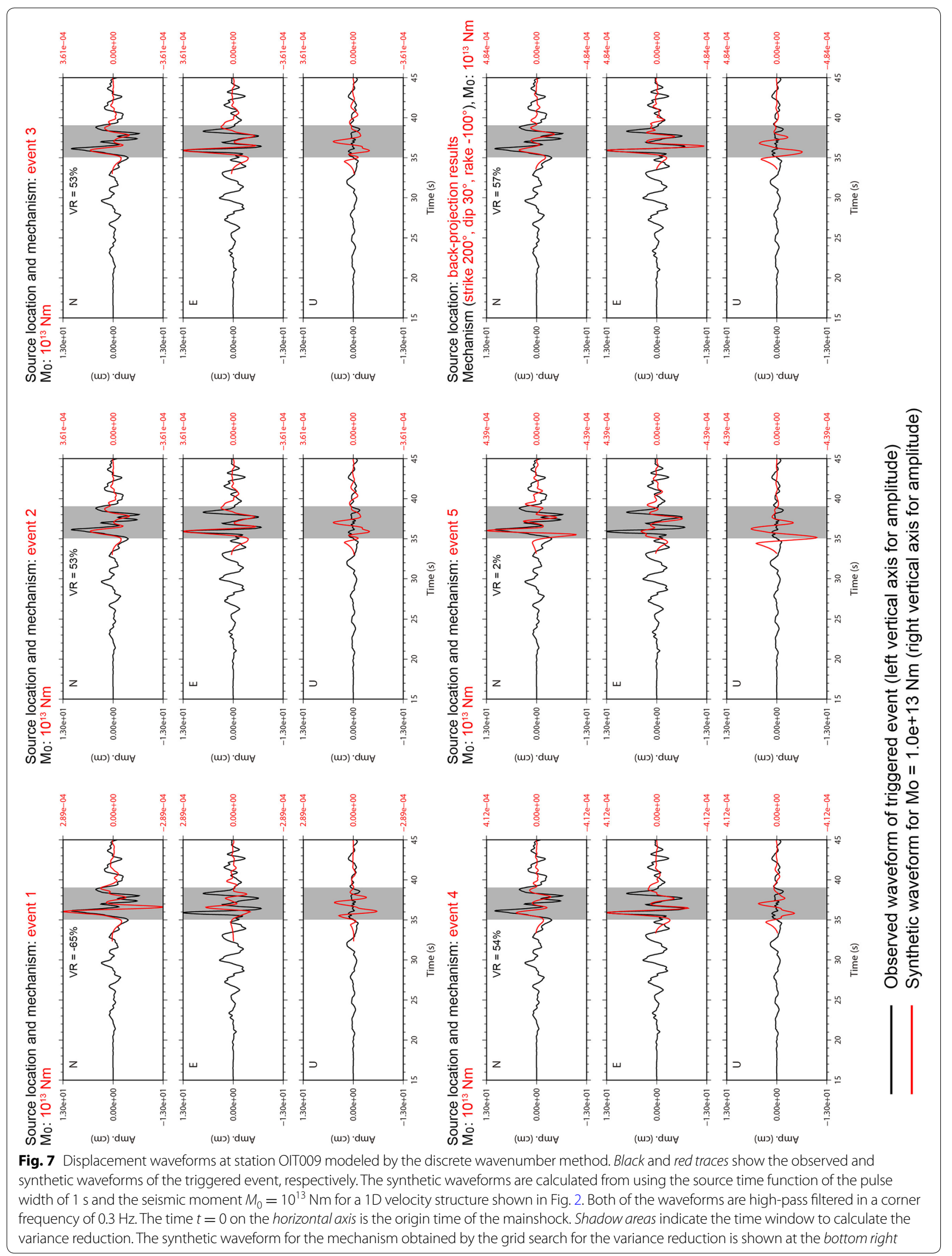




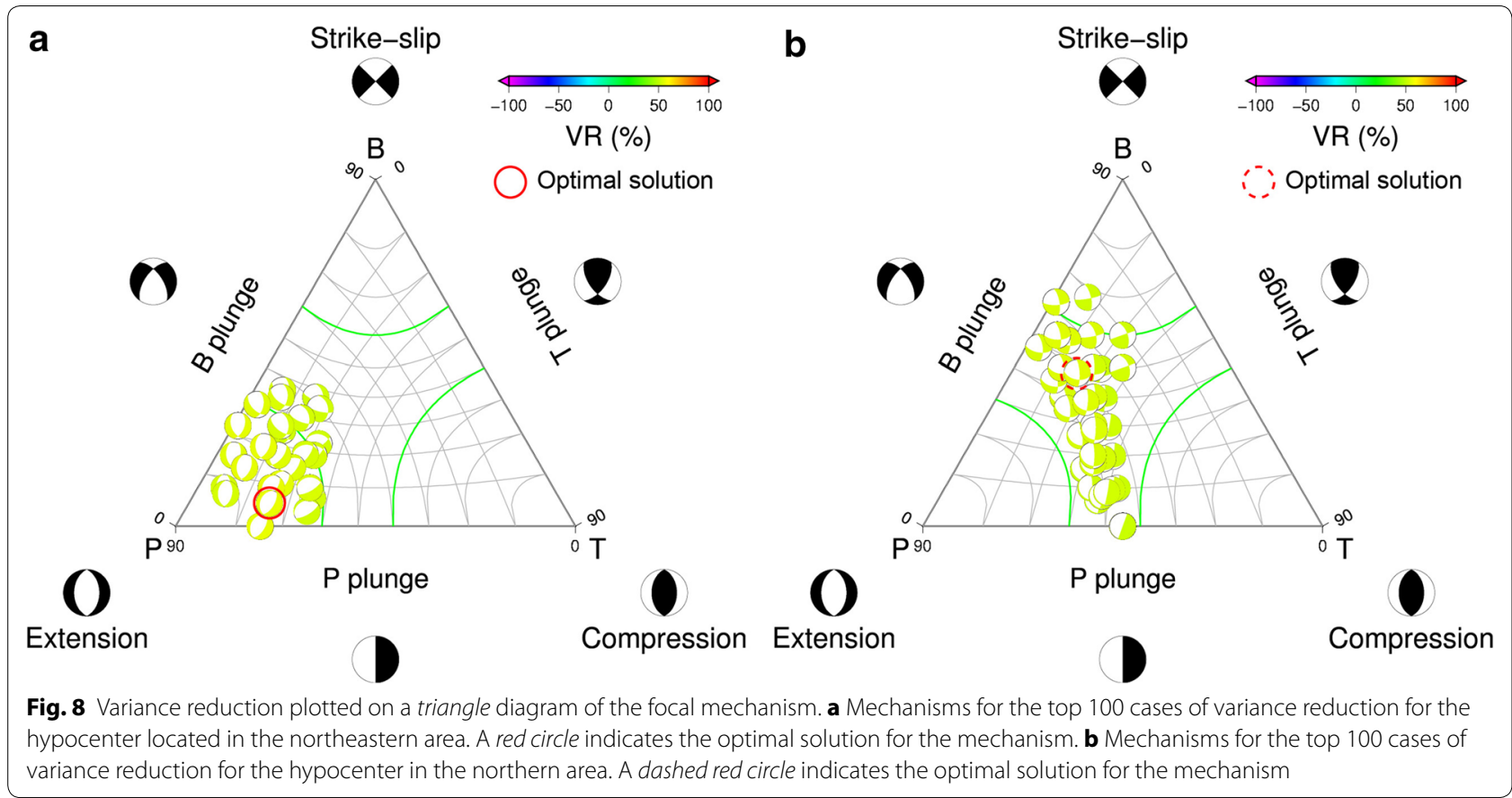

the triggered event. Synthetic waveforms for a normalfault mechanism and a normal-fault with strike-slip components at the estimated source locations also explain the observed waveforms. Although our approach does not constrain the strike direction well, the results of our waveform analysis indicate that the triggered earthquake occurred near the station that observed the strong motions, primarily by a normal-fault mechanism or a normal-fault with strike-slip components.

\section{Additional file}

Additional file 1. This file includes additional figures (Figures S1-3) and a table (Table S1).

\section{Abbreviations}

AQUA: accurate and quick analysis system for source parameters; CMT: centroid moment tensor; JMA: Japan Meteorological Agency; JSPS: Japan Society for the Promotion of Science; MT: moment tensor; NIED: National Research Institute for Earth Science and Disaster Resilience.

\section{Authors' contributions}

TN conducted the data analysis and the calculation of seismic waveforms and wrote the paper. SA coordinated the strong-motion network data and contributed to the data analysis and the calculation. Both authors read and approved the final manuscript.

\section{Acknowledgements}

Discussions with Hisahiko Kubo, Wataru Suzuki, and Nelson Pulido were very fruitful. Constructive comments and suggestions from two anonymous reviewers were very helpful. We used the hypocenter catalogue provided by the Japan Meteorological Agency (JMA). We used data from K-NET and
KiK-net, operated by the National Research Institute for Earth Science and Disaster Resilience (NIED). This work was supported by JSPS KAKENHI Grant Number 26282105.

\section{Competing interests}

The authors declare that they have no competing interests.

Received: 1 August 2016 Accepted: 9 December 2016

Published online: 03 January 2017

\section{References}

Aoi S, Kunugi T, Suzuki W, Kubo H, Morikawa N, Fujiwara H (2016) Strong motion and source processes of the 2016 Kumamoto earthquake sequence. In: 2016 Japan Geoscience Union Meeting MIS34-06

Frohlich C (1992) Triangle diagrams: ternary graphs to display similarity and diversity of earthquake focal mechanisms. Phys Earth Planet Int 75(1-3):193-198. doi:10.1016/0031-9201(92)90130-N

Hartzell SH (1978) Earthquake aftershock as Green's function. Geophys Res Lett 5:1-4. doi:10.1029/GL005i001 p00001

Ishii M, Shearer PM, Houston H, Vidale JE (2005) Extent, duration and speed of the 2004 Sumatra-Andaman earthquake imaged by the Hi-net array. Nature 435:933-936. doi:10.1038/nature03675

Kamata H (1989) Volcanic and structural history of the Hohi volcanic zone central Kyushu, Japan. Bull Volcanol 51(5):315-332. doi:10.1007/ BF01056894

Kao H, Shan SJ (2004) The source-scanning algorithm: mapping the distribution of seismic sources in time and space. Geophys J Int 157(2):589-594. doi:10.1111/j.1365-246X.2004.02276.X

Kikuchi M, Kanamori H (1991) Inversion of complex body waves-III. Bull Seismol Soc Am 81 (6):2335-2350

Koketsu K, Miyake H, Fujiwara H, Hashimoto T (2008) Progress towards a Japan integrated velocity structure model and long-period ground motion hazard map. In: Proceedings 14th World Conference Earthquake Engineering S10-038

Kubo H, Suzuki W, Aoi S, Sekiguchi H (2016) Source rupture process of the 2016 Kumamoto, Japan, earthquakes estimated from 
strong-motion waveforms. Earth Planets Space 68:161. doi:1 0.1186/ s40623-016-0536-8

Matsumura M, Ito Y, Kimura H, Obara K, Sekiguchi S, Hori S, Kasahara K (2006) Development of accurate and quick analysis system for source parameters (AQUA). Zisin 2(59):167-184

Nakamura T, Takenaka H (2006) A numerical analysis of seismic waves for an anisotropic fault zone. Earth Planets Space 58:569-582. doi:10.1186/ BF03351954

Okada Y, Kasahara K, Hori S, Obara K, Sekiguchi S, Fujiwara H, Yamamoto A (2004) Recent progress of seismic observation networks in JapanHi-net, F-net, K-NET and KiK-net. Earth Planets Space 56:XV-XXVIII. doi:10.1186/BF03353076

Ozawa T, Fujita E, Ueda H (2016) Crustal deformation associated with the 2016 Kumamoto Earthquake and its effect on the magma system of Aso volcano. Earth Planets Space 68:186. doi:10.1186/s40623-016-0563-5
Spudich P, Cranswick E (1984) Direct observation of rupture propagation during the 1979 Imperial Valley earthquake using a short baseline accelerometer array. Bull Seismol Soc Am 74(6):2083-2114

Spudich P, Frazer LN (1984) Use of ray theory to calculate high-frequency radiation from earthquake sources having spatially variable rupture velocity and stress drop. Bull Seismol Soc Am 74(6):2061-2082

Vlček J, Fischer T, Vilhelm J (2015) Back-projection stacking of P-and S-waves to determine location and focal mechanism of microseismic events recorded by a surface array. Geophys Prospect 64(6):1428-1440. doi:10.1111/1365-2478.12349

Yano TE, Matsubara M (2016) The significance of seismicity after the 2016 Kumamoto Earthquake sequence. In: 2016 Japan Geoscience Union Meeting MIS34-P05 